\title{
External Electric Field Effect on Electrons Transport in Carbon Nanotubes
}

\author{
Sulemana S. Abukari ${ }^{{ }^{*}}$, Samuel Y. Mensah ${ }^{1}$, Musah Rabiu ${ }^{2}$, Kofi W. Adu ${ }^{3,4}$, Natalia G. Mensah ${ }^{5}$, \\ Kwadwo A. Dompreh ${ }^{1}$, Anthony Twum ${ }^{1}$, Matthew Amekpewu ${ }^{2}$
} ${ }^{1}$ Department of Physics, Laser and Fibre Optics Centre, University of Cape Coast, Cape Coast, Ghana; ${ }^{2}$ Department of Applied
Physics, University for Development Studies, Navorongo, Ghana; ${ }^{3}$ Department of Physics, The Pennsylvania State University, Al-
toona, USA; ${ }^{4}$ Materials Research Institute, The Pennsylvania State University, University Park, USA; ${ }^{5}$ Department of Mathematics,
University of Cape Coast, Cape Coast, Ghana.

Email: "sulemana70@gmail.com

Received July $1^{\text {st }}, 2013$; revised August $11^{\text {th }}, 2013$; accepted August $25^{\text {th }}, 2013$

Copyright (C) 2013 Sulemana S. Abukari et al. This is an open access article distributed under the Creative Commons Attribution License, which permits unrestricted use, distribution, and reproduction in any medium, provided the original work is properly cited.

\begin{abstract}
We consider a simple model of carbon nanotubes (CNTs) subject to external electric field $E(t)$. Using a tight-binding approximation for the description of energy bands of CNTs, together with the standard Boltzmann transport equation and constant relaxation time, we predict the effect of self-induced transparency and absolute negative conductivity. The predicted effects may be useful in diagnostics of carbon nanotubes as well as in the amplification and efficiency conversion of electromagnetic signals.
\end{abstract}

Keywords: Carbon Nanotubes; Electric Field; Electric Current Density; Negative Differential Conductivity

\section{Introduction}

The electrical transport properties of carbon nanotubes (CNs) have been the subject of much research ever since the discovery by Iijima [1] of the quasi-one-dimensional monomolecular structures. This may be due to their abilities to exhibit Bloch oscillations [2-5] at moderate electric field strengths. This oscillatory response makes CNs inherently nonlinear and as such can perform varieties of transport phenomena. Under different conditions of an external electric field, an electron is predicted to reveal a variety of physical effects such as Bloch oscillations, self-induced transparency, negative differential conductivity, absolute negative conductance [4], etc.

In this paper we study theoretically two phenomena in CNs, which are self-induced transparency and absolute negative conductance for the following cases respectively:

1) When the CNs is exposed to an a. c. electric field i.e. $E(t)=E_{1} \cos \omega t$.

2) When the CNs is exposed to an a. c. and d.c electric field i.e. $E(t)=E_{o}+E_{1} \cos \omega t$.

*Corresponding author.

\section{Theory}

Following references $[3,4]$ and using the approach similar to reference [6], we consider a response of electrons in an undoped achiral single-wall carbon nanotubes subject to an external electric field.

$$
E(t)=E_{o}+E_{1} \cos \omega t
$$

We use the semiclassical approximation in which $\pi$ electrons are considered as classical particles with dispersion law extracted from the quantum theory in the tight-binding approximation [4].

Considering the hexagonal crystalline structure of zigzag and armchair CNTs within tight binding approximation, the dispersion relation is given as [4] respectively,

$$
\begin{aligned}
\varepsilon_{s}\left(p_{z}\right)= & \pm \gamma_{0}\left[1+4 \cos \left(a p_{z}\right) \cos \left(\frac{a}{\sqrt{3}} s \Delta p_{\varphi}\right)\right. \\
& \left.+4 \cos ^{2}\left(\frac{a}{\sqrt{3}} s \Delta p_{\varphi}\right)\right]^{1 / 2} \\
\varepsilon_{s}\left(p_{z}\right)= & \pm \gamma_{0}\left[1+4 \cos \left(a s \Delta p_{\varphi}\right) \cos \left(\frac{a}{\sqrt{3}} p_{z}\right)\right.
\end{aligned}
$$




$$
\left.+4 \cos ^{2}\left(\frac{a}{\sqrt{3}} p_{z}\right)\right]^{1 / 2}
$$

here $\gamma_{0} \sim 3.0 \mathrm{eV}$ is the overlapping integral, $p_{z}$ is the axial component of quasimomentum, $\Delta p_{\varphi}$ is transverse quasimomentum level spacing and $s$ is an integer. The expression for $a$ in Equation (2) is given as $a=3 b / 2 \hbar$ with the C-C bond length $b=0.142 \mathrm{~nm}$ and $\hbar$ is the Plank's constant, we shall assume $\hbar=1$. The - and + signs correspond to the valence and conduction bands, respectively. Due to the transverse quantization of the quasi-momentum, its transverse component can take $n$ discrete values, $p_{\varphi}=s \Delta p_{\varphi}=\pi \sqrt{3} s / a n(s=1, \cdots, n)$. Unlike transverse quasimomentum $p_{\varphi}$, the axial quasimomentum $p_{z}$ is assumed to vary continuously within the range $0 \leq p_{z} \leq 2 \pi / a$, which corresponds to the model of infinitely long $\operatorname{CNT}(L=\infty)$. This model is applicable to the case under consideration because of the restriction to the temperatures and/or voltages well above the level spacing [4]. We assume that there is no inter band transition or transition between different states i.e.

$k_{B} T>\varepsilon_{C}, \Delta \varepsilon$, where $k_{B}$ is Boltzmann constant, $T$ is the temperature, $\varepsilon_{C}$ is the charging energy. The energy level spacing $\Delta \varepsilon$ is given by $\Delta \varepsilon=\frac{\pi \hbar v_{F}}{L}$, where $v_{F}$ is the Fermi velocity and $L$ is the carbon nanotube length [6].

Proceeding as in 4, we employ Boltzmann equation with a constant relaxation time

$$
\frac{\partial f(p, t)}{\partial t}+e E(t) \frac{\partial f(p)}{\partial p}=-\frac{\left[f_{s}-f_{0}(p)\right]}{\tau}
$$

where $e$ is the electron charge, $f_{0}(p)$ is the equilibrium distribution function, $f(p, t)$ is the distribution function, and $\tau$ is the relaxation time. The electric field $E(t)$ is applied along CNTs axis. In this problem the relaxation term $\tau$ is assumed to be constant. The justification for being constant can be found in [3]. The relaxation term of Equation (3) describes the effects of the dominant type of scattering (e.g. electron-phonon and electron-twistons) [3]. We have noted that the distribution function $f(p)$ is periodic with a period $2 \pi / a$.

Expanding the distribution functions of interest in Fourier series as;

$$
f_{0}(p)=\Delta p_{\varphi} \sum_{s=1}^{n} \delta\left(p_{\varphi}-s \Delta p_{\varphi}\right) \sum_{r \neq 0} f_{r s} \mathrm{e}^{i a r p_{z}}
$$

and

$$
f(p, t)=\Delta p_{\varphi} \sum_{s=1}^{n} \delta\left(p_{\varphi}-s \Delta p_{\varphi}\right) \sum_{r \neq 0} f_{r s} \mathrm{e}^{i a r p_{z}} \varnothing_{\nu}(t)
$$

here the coefficient, $\delta(x)$ is the Dirac delta function, $f_{r s}$ is the coefficient of the Fourier series and $\varnothing_{v}(t)$ is the factor by which the Fourier transform of the nonequilibrium distribution function differs from its equilibrium distribution counterpart. The equilibrium distribution function $f_{o}(p)$ can be expanded in the analogous series with coefficients as follows

$$
f_{r s}=\frac{a}{2 \pi} \int_{0}^{\frac{2 \pi}{a}} \frac{\mathrm{e}^{-i a r p_{z}}}{1+\exp \left(\varepsilon_{s}\left(p_{z}\right) / k_{B} T\right)} \mathrm{d} p_{z}
$$

Substituting Equations (5) and (6) into Equation (4), and solving with Equation (1) we obtain

$$
\varnothing_{\nu}(t)=\sum_{k=-\infty}^{\infty} \sum_{m=-\infty}^{\infty} \frac{J_{k}(r \beta) J_{k-v}(r \beta)}{1+i\left(e a r E_{0}+k \omega\right) \tau} \exp (i v \omega t)
$$

where $\beta=\frac{e a E_{1}}{\omega}, J_{k}(r \beta)$ is the Bessel function of the $k^{\text {th }}$ order and $\Omega=e a E_{0}$ for zigzag CNTs and $\Omega=e a E_{0} / \sqrt{3}$ for armchair CNTs.

We determine the surface current density as

$$
=\frac{2 e}{(2 \pi \hbar)^{2}} \iint f(p) v_{z}(p) \mathrm{d}^{2} p
$$

or

$$
j_{z}=\frac{2 e}{(2 \pi \hbar)^{2}} \sum_{s=1}^{n} \int_{0}^{\frac{2 \pi}{a}} f\left(p_{z}, s \Delta p_{\varphi}, \varnothing_{v}(t)\right) v_{z}(p) \mathrm{d} p_{z}
$$

and the integration is carried over the first Brillouin zone. We consider the relation $v_{z}\left(p_{z}, s \Delta p_{\varphi}\right)=\partial \varepsilon_{r s}\left(p_{z}\right) / \partial p_{z}$, and represent $\varepsilon_{s}\left(p_{z}\right) / \gamma_{o}$ in Fourier series with the coefficients defined similar to Equation (7).

Substituting Equations (6) and (8) into (9) we find the current density for the CNs after averaging over a period of time $t$, as

$$
j_{z}=\frac{8 e \gamma_{0}}{\sqrt{3 \hbar n a}} \sum_{r=1}^{\infty} r \sum_{s=1}^{n} f_{r s} \varepsilon_{r s} \sum_{k=-\infty}^{\infty} \frac{J_{k}^{2}(r \beta)(\Omega r+k \omega) \tau}{1+((\Omega r \tau+k \omega) \tau)^{2}}(10)
$$

From Equation (10) we obtain

$$
j_{z}=\frac{8 e \gamma_{0}}{\sqrt{3 \hbar n a_{c-c}}} \times \sum_{r=1}^{\infty} r\left[\frac{J_{0}^{2}(r \beta)}{1+(\Omega r \tau)^{2}}+2 \sum_{k=1}^{\infty} \frac{J_{k}^{2}(r \beta)\left[1+(\Omega r \tau)^{2}-(k \omega \tau)^{2}\right]}{\left[1+(\Omega r \tau-k \omega \tau)^{2}\right]\left[1+(\Omega r \tau+k \omega \tau)^{2}\right]}\right] \times \Omega r \tau \sum_{s=1}^{n} f_{r s} \varepsilon_{r s}
$$


If $k \omega \tau \gg \Omega \tau$ and $\omega \tau \gg 1$, Equation (11) takes the form

$$
\begin{aligned}
& j_{z}=\frac{8 e \gamma_{0}}{\sqrt{3 \hbar n a_{c-c}}} \\
& \times \sum_{r=1}^{\infty} r\left[\frac{J_{0}^{2}(r \beta)}{1+(\Omega \tau r)^{2}}-\frac{2}{(\omega \tau)^{2}} \sum_{k=1}^{\infty} \frac{J_{k}^{2}(r \beta)}{(k)^{2}}\right] \Omega r \tau \sum_{s=1}^{n} f_{r s} \varepsilon_{r s}
\end{aligned}
$$

where $r \beta=\left(e a r E_{1}\right) / \omega, J_{0}(r \beta)$ is the Bessel function of the zeroth order.

From Equation (12) it is seen that near the zeros of $J_{0}(r \beta)$ and $k \omega \tau \gg 1$, the CNTs exhibit absolute negative resistance in the transparency regions. That is, the low frequency oscillations are amplified in these regions. This phenomenon is called absolute negative conductivity and was first observed by Kryuchkov et al. [6].

In the limiting case of $\omega \tau \gg 1, E_{0}=0$, we find that the current density for the CNTs is

$$
j_{z}=\frac{8 e \gamma_{0}}{\sqrt{3 \hbar n a_{c-c}}} \sum_{r=1}^{\infty} r J_{0}(r \beta) \sin (r \beta \sin \omega t) \sum_{s=1}^{n} f_{r s} \varepsilon_{r s}
$$

From Equation (13) at each zero of the $J_{0}(r \beta)$ function the current density vanishes and the CNTs become transparent. See also Ignatov et al. [7].

\section{Results and Discussion}

Equations (12) and (13) describe the theory of electron transport in the presence of an external electric field $E(t)$. We assume that the equations do not take into account both interband transitions and quantum-mechanical corrections to the intraband motion or resonant transitions inherent in the $\mathrm{CN}$ lattice. To estimate the restriction on the fundamental frequency necessary for resonant transitions to be negligible, we apply a general approximate relation for the electron state density in a CNT $[3,8]$ that establishes the low-frequency edge $\bar{\omega}$ of the optical transition band in metallic CNTs; $\left(\bar{\omega}<3 \gamma_{o} b\right) / 2 \hbar R$, where $R$ is the CNTs radius. This condition is decisive for theapplicability of the developed theory in CNTs. As the CNTs radius increases, the upper limit for the fundamental frequency is shifted to the red. Thus, CNs with not too large radii are of the most interest. Another limitation is related to the strength of the driving field where interband tunneling is negligible: the Stark frequency corresponding to this field must be smaller than the conduction zone bandwidth $E_{1}<\gamma_{o} b$ [9]. Otherwise, the bond of $\pi$-electrons with the crystalline lattice breaks down, resulting in a strong Stark broadening and making the dispersion law ((2) and (3)) inapplicable. For the condition of high-frequency fields $E(t)=E_{1} \cos \omega t$, an analytical expression for the current density was obtained in Equation (13). Figures 1 and 2 illustrate the graphs of $j_{z} / j_{0}$ on $r \beta$ for $(12,0)$ metallic zigzag and $(10,10)$

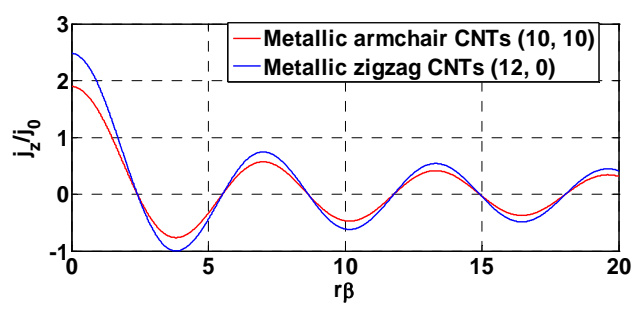

Figure 1. Normalised current density $\left(j_{z} / j_{0}\right)$ as a function of the ac amplitude $(r \beta)$ for expression (13); metallic armchair (red) and metallic zigzag (blue).

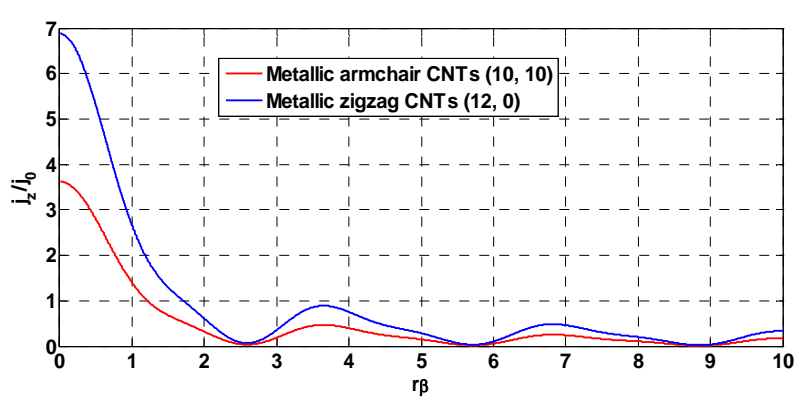

Figure 2. Normalised current density $\left(j_{z} / j_{0}\right)$ as a function of the ac amplitude $(r \beta)$ for expression (12); metallic armchair (red) and metallic zigzag (blue).

metallic armchair CNTs calculated by Equation (13). From the figure it is seen that the amplitude of the current density oscillates with increasing amplitude of the high frequency and in the zeros of the $J_{o}(r \beta)$ function or when $r \beta$ is equal to the roots of the zeroth order Bessel function $(2.4,4.8,8.4,11.8,14.8,18.0)$ the total current is zero (the self-transparency effect). This effect is due to the non-parabolicity of the electron energy band which is stronger in the CNTs. However, when the CNTs is exposed to an a.c. and d.c electric fields, i.e. $E(t)=$ $E_{o}+E_{1} \cos \omega t$ for $k \omega \tau \gg \Omega \tau$ and $\omega \tau \gg 1$, we obtained expression (12) which is an indication for absolute negative conductivity i.e. current flows against the applied d.c field. See Figure 2. Figure 2 elucidates the dependence of $j_{z} / j_{0}$ on $r \beta$ for $(12,0)$ metallic zigzag and $(10$, 10) metallic armchair CNTs obtained via the calculation of Equation (12). In the low limit of $(r \beta)$, we observed that as $r \beta$ increases, the normalized current $j_{z} / j_{0}$ decreases and at a certain value e.g., $r \beta=r \beta_{\min }$ the normalized current reaches a minimum value $\left(j_{z} / j_{0}\right)_{\min }$. Thus, we predict the region with absolute negative conductivity (ANC). Also we note that such state will be unstable and will lead to CNTs segmentation into domains. The region of absolute negative conductivity decreases when external electric field increases. We noted that due to the high density of states of conduction electrons in carbon nanotubes and the specific dispersion law inherent in hexagonal crystalline structure result in a uniquely higher current densities for the metallic zigzag CNTs than the me- 
tallic armchair CNTs.

\section{Conclusion}

In conclusion, using the solution to the Boltzmanns transport equation with constant relaxation time, we report on a theoretical analysis of a simple model of carbon nanotubes (CNTs) subject to an external electric field. We predict the effect self-induced transparency and absolute negative conductivity. The predicted effects may be useful in diagnostics of carbon nanotubes as well as in the amplification and efficiency conversion of electromagnetic signals. We noted that the metallic zigzag CNT has higher current density than the metallic armchair CNT. This is attributed to the fact that the metallic zigzag CNT has higher density of states of conduction electrons than the metallic armchair CNT. Another reason for the metallic ziagzag CNT to exhibit higher current density than the armchair is due to its dispersion law which is derived from the hexagonal structure.

\section{REFERENCES}

[1] S. Iijima, "Helical Microtubules of Graphitic Carbon," Nature, Vol. 354, 1991, pp. 56-58. http://dx.doi.org/10.1038/354056a0

[2] O. M. Yevtushenko, G. Ya. Slepyan, S. A. Maksimenko, A. Lakhtakia and D. A. Romanov, "Nonlinear Electron Transport Effects in a Chiral Carbon Nanotube," Physical Review Letters, Vol. 79, No. 6, 1997, pp. 1102-1105. http://dx.doi.org/10.1103/PhysRevLett.79.1102
[3] G. Ya. Slepyan, S. A. Maksimenko, A. Lakhtakia, O. M. Yevtushenko and A. V. Gusakov, "Electronic and Electromagnetic Properties of Nanotubes," Physical Review B, Vol. 57, No. 16, 1998, pp. 9485-9497. http://dx.doi.org/10.1103/PhysRevB.57.9485

[4] S. A. Maksimenko and G. Ya. Slepyan, "Negative Differential Conductivity in Carbon Nanotubes," Physical Review Letters, Vol. 84, No. 2, 2000, pp. 362-365. http://dx.doi.org/10.1103/PhysRevLett.84.362

[5] S. S. Abukari, S. Y. Mensah, N. G. Mensah, K. A. Dompreh, A. Twum and F. K. A. Allotey, "Rectification Due to Harmonic Mixing of Two Coherent Electromagnetic Waves with Commensurate Frequencies in Carbon Nanotubes," European Physical Journal B, Vol. 86, 2013, p. 106. http://dx.doi.org/10.1140/epib/e2013-30011-3

[6] S. V. Kryuchkov and V. A. Yakovlev, "Electron-Phonon Mechanism of the Self-Induced Transparency of Semiconductors," Fizika Tekhn. Poluprov, Vol. 9, No. 2, 1975, pp. 363-365.

[7] A. A. Ignatov and Yu. A. Romanov, "Romanov SelfInduced Transparency in Semiconductors Superlattices," Fizika Tverdogo Tela, Soviet Physics, Solid State, Vol. 17, 1975, p. 3387.

[8] J. W. Mintmire and C. T. White, "Universal Density of States for Carbon Nanotubes," Physical Review Letters, Vol. 81, No. 12, 1998, pp. 2506-2509. http://dx.doi.org/10.1103/PhysRevLett.81.2506

[9] F. G. Bass and A. A. Bulgakov, "Kinetic and Electrodynamic Phenomena in Classical and Quantum Semiconductor Superlattices," Nova Science Publishers, New York, 1997. 